\title{
Distinct gene expression profiles associated with the susceptibility of pathogen-specific CD4+ T cells to HIV-1 infection
}

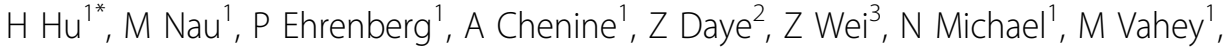 \\ J Kim', M Marovich', S Ratto-Kim ${ }^{1}$
}

From AIDS Vaccine 2012

Boston, MA, USA. 9-12 September 2012

\section{Background}

HIV infection causes the progressive depletion of CD4+ $\mathrm{T}$ cells. Contrary to the early loss of CD4 response to opportunistic pathogens like Candida albicans, cytomegalovirus (CMV)-specific CD4 response is persistent when total $\mathrm{CD} 4+\mathrm{T}$ cell number is low. The mechanism is less clear. Despite considerable knowledge for the impact of HIV infection on total CD4+ T cells and their subsets, little is known about HIV infection of CD4+ $\mathrm{T}$ cells of different pathogen/antigen (Ag) specificity.

\section{Methods}

PBMC from HIV-negative donors were CFSE-labeled and stimulated ex vivo with pathogen-specific antigens including viral (CMV), bacterial (Tetanus Toxoid: TT) and fungal (Candida albicans) antigens. HIV infection of Ag-specific CD4+ T cells was determined by intracellular p24 production in CFSE-low population.

\section{Results}

While TT- and Candida-specific CD4+ T cells were permissive, CMV-specific CD4+ T cells are highly resistant to both X4 and R5 HIV independent of coreceptor useage. Quantification of HIV DNA in sorted, antigenspecific CD4+ $\mathrm{T}$ cells demonstrated a reduction of both strong-stop and full-length HIV DNA in CMV-specific CD4+ T cells. $\beta$-chemokine neutralization enhanced HIV entry and viral replication in TT- and Candidaspecific CD4+ T cells, whereas HIV infection in CMVspecific $\mathrm{CD} 4+\mathrm{T}$ cells remained low despite increased HIV entry by $\beta$-chemokine neutralization, suggesting both entry and post-entry HIV restriction in CMVspecific cells. Microarray analysis revealed distinct gene expression profiles that involved selective upregulation of a broad array of antiviral genes in CMV-specific CD4+ T cells, whereas TT- and Candida-specific CD4+ $\mathrm{T}$ cells mainly upregulated a Th17 inflammatory response.

\section{Conclusion}

Our data suggest a mechanism for the persistence of CMV-specific CD4 response and the earlier loss of mucosal Th17-associated TT- and Candida-specific CD4 response in AIDS patients. The model described is useful in HIV vaccine studies by evaluating the susceptibility of vaccine-specific CD4 responses to HIV infection.

\section{Author details}

${ }^{1}$ U.S. Military HIV Research Program, Silver Spring, MD, USA. ${ }^{2}$ University of Pennsylvania School of Medicine, Philadelphia, PA, USA. ${ }^{3}$ New Jersey Institute of Technology, Newark, NJ, USA.

Published: 13 September 2012

doi:10.1186/1742-4690-9-S2-046

Cite this article as: Hu et al: Distinct gene expression profiles

associated with the susceptibility of pathogen-specific CD4+ T cells to HIV-1 infection. Retrovirology 2012 9(Suppl 2):O46.

'U.S. Military HIV Research Program, Silver Spring, MD, USA

Full list of author information is available at the end of the article

(C) 2012 Hu et al; licensee BioMed Central Ltd. This is an Open Access article distributed under the terms of the Creative Commons 